\title{
ADAPTACIÓN DE LA DOCENCIA POR COVID-19: EXPERIENCIA EN LA INGENIERÍA AUTOMÁTICA
}

\author{
Oscar Miguel-Escrig, Carlos Díaz-Sanahuja, Ignacio Peñarrocha-Alós, Roberto Sanchis-Llopis \\ Departmento de Ingeniería de Sistemas y Diseño. Universitat Jaume I \\ Campus del Riu Sec. Av. Vicent Sos Baynat s/n. \\ CP 12071 Castelló de la Plana. España \\ e-mail: \{omiguel,csanahuj,ipenarro,rsanchis\}@uji.es
}

\begin{abstract}
Resumen
En este trabajo se describe y analiza la experiencia docente relativa al curso 2020-2021 en la asignatura Sistemas Automáticos. Debido a los efectos de la pandemia, se ha tenido que adaptar la docencia al no disponer de aulas suficientes que permitan una temporización correcta de los contenidos. Por lo tanto, se han desarrollado nuevos contenidos, mayoritariamente en forma de vídeo, que permiten el seguimiento del curso. Con los registros de actividad de estos nuevos recursos se ha observado la efectividad del método empleado así como su validez comparándolo con resultados de años anteriores.
\end{abstract}

Palabras clave: Covid-19, Educación en ingeniería, vídeos

\section{INTRODUCCIÓN}

Con el inicio de la expansión de la covid-19 a principios del año 2020 el mundo ha sufrido un cambio sin precedentes que ha afectado a todas las facetas de la vida. Este cambio se concretó en España a mediados de marzo de 2020 con el establecimiento del estado de alarma, afectando a la movilidad y a la agrupación de personas, lo cual repercutió, entre otros ámbitos, a la docencia.

Este cambio de paradigma afectó a todos los integrantes de la comunidad universitaria alrededor del mundo por la gran incertidumbre que plantea $[8,13]$. No obstante, las universidades optaron de forma unánime por adaptar su docencia presencial a una docencia en línea [14], con las dificultades que ello planteaba. Estudios como [12, 27] reflejan el impacto de dicha transición, que en algunos casos resulta apresurada o incompleta por falta de recursos.

Pese a que algunos estudios indican que los estudiantes perciben una mejor transferencia de conocimiento en sesiones presenciales, también reseñan que la modalidad en linea resulta útil y necesaria dadas las circunstancias [5]. Este hecho no entra en conflicto con la metodología didáctica en sí, ya que la docencia en linea ha sido ampliamente es- tudiada y ofrece también grandes resultados [15]. Algunos autores la consideran un reemplazo viable a la docencia presencial [17, 18], e instituciones como la UNED, que imparte su docencia en línea, avalan su eficacia.

Por lo tanto, el problema en la transición a la docencia en línea que ponen de manifiesto los diferentes trabajos mencionados reside en una incorrecta planificación de la docencia. Existen diferentes vertientes en cuanto a cómo organizar y secuenciar los contenidos. Propuestas como el análisis de contenido [3, 20] permiten organizar la materia en un orden lógico según los conceptos clave y su relación entre ellos. Otras como el análisis de tareas $[11,10]$ están más orientadas a organizar el contenido procedimental, como la resolución de problemas. Reigeluth [21] propone una perspectiva que integra a las dos anteriores, planteando en primer lugar los conceptos generales, entrando en detalle en ellos y sus técnicas, para luego relacionarlos y enmarcarlos dentro de los contenidos a abordar.

Además de organizar y secuenciar los contenidos según los preceptos de alguna de las teorías anteriores, la situación actual requiere del diseño y desarrollo de materiales que se adecuen a la docencia en línea. Entre las técnicas más comunes se encuentran las videoconferencias como elemento de docencia síncrona, las cuales han demostrado ofrecer resultados similares a la docencia presencial $[7,25]$. Con carácter asíncrono encontramos las lecciones grabadas o cualquier tipo de vídeo docente.

De acuerdo con la teoría del cognitivismo aplicada al aprendizaje multimedia, todas las formas de transmitir conocimiento, mediante vista u oído, son capaces de activar nuestra estructura cognitiva, siendo el aprendizaje más efectivo cuando la información es transmitida por más de un canal $[16,22]$, es decir, se obtienen mejores resultados de aprendizaje usando un vídeo en el cual imagen y sonido transmiten un mensaje complementario simultáneamente, que si solo se usa un podcast o un texto como método de transmisión del mensaje.

Por otra parte, el uso de vídeos dota al alumnado 
de cierto grado de control sobre su propio aprendizaje, lo cual según ciertos estudios incentiva su motivación y les permite regular su carga cognitiva $[1,26]$, y además, reduce la percepción de la carga cognitiva usada en el aprendizaje [24].

Sin embargo, si bien es típicamente aceptado que el grado de aprendizaje es directamente proporcional a la interacción del alumno con la materia $[4,6]$, el visionado de un vídeo, de naturaleza pasiva, también aporta cierto grado de interacción, permitiendo pausar, revisionar y tomar notas al respecto, aportando grandes beneficios al aprendizaje [19].

Teniendo esto en cuenta, en este trabajo se presenta una experiencia educativa en la cual se ha replanteado parte de la docencia a consecuencia de la coyuntura pandémica actual. Debido a las restricciones, la docencia de parte de las prácticas en laboratorio se ha sustituido por recursos disponibles en línea, bajo la forma de vídeos, que permiten al alumnado una formación autónoma. Estos vídeos no pretenden sustituir completamente a los recursos usados hasta la fecha, sino complementar la enseñanza como material de apoyo auxiliar.

\section{PRESENTACIÓN DEL CASO}

En este trabajo se presenta la experiencia docente de la asignatura "Sistemas Automáticos". Se trata de una asignatura impartida en el primer semestre del tercer curso transversal a varios grados de ingeniería de la rama industrial, y tiene especial importancia ya que en algunos de estos grados no se vuelven a tratar temas relacionados con el control y la automatización.

Los contenidos de la asignatura comprenden muchos aspectos del control, abarcando desde el modelado e identificación de sistemas, hasta el diseño de controladores y análisis de sistemas realimentados. Por ello la asignatura se estructura en unidades temáticas tratando cada uno de estos aspectos. Si bien son contenidos con entidad propia, cabe destacar que en la práctica son necesarios conocimientos de cada unidad temática para desarrollar una aplicación de control funcional.

La metodología didáctica de la asignatura se estructura en cuatro actividades formativas: 1) sesiones de teoría, bajo la forma de lección magistral; 2) sesiones prácticas de problemas, más participativas o guiadas dependiendo de las características del temario; 3) sesiones prácticas de laboratorio, en que se aplican los conocimientos de la unidad temática, requiriendo en su caso trabajo previo; y 4) trabajo personal, en forma de problemas entregables, cuestionarios o trabajos previos.
En cuanto a la secuenciación de los contenidos, estos se impartían por unidad temática siguiendo el orden teoría-problemas-prácticas, requiriendo trabajo autónomo entre las sesiones en forma de problemas. Sin embargo, debido a la situación de pandemia, la temporización original se ha visto modificada como consecuencia de la reducción del aforo máximo de las aulas, y por tanto, su disponibilidad.

Esto ha causado que el lapso temporal entre sesiones que permitía en años anteriores al alumnado desarrollar el trabajo personal (entregables, cuestionarios y previos) se haya reducido tanto que la secuenciación anterior ya no es sostenible. En particular, la reducción ha sido especialmente significativa en el lapso entre problemas y prácticas de laboratorio, siendo las actividades autónomas que se realizaban en este espacio temporal las más afectadas.

\section{MODIFICACIONES Y SEGUIMIENTO}

De acuerdo con lo expuesto en la sección anterior queda claro que las modificaciones debían aplicarse principalmente a las tareas que desarrollaban los estudiantes de forma autónoma entre las sesiones de problemas y prácticas. Se dá además la circunstancia de que este trabajo autónomo estaba muy vinculado con la sesión de prácticas posterior y en algunos casos constituía un trabajo previo para la misma.

Por lo tanto, los materiales docentes empleados para estas actividades han sido modificados, distribuyéndose entre dos actividades con entidad propia: Entregables de Problemas (EP) y Trabajos Previos Recomendables (TPR). De esta forma los EP son trabajos autónomos a realizar después de la sesión de problemas, pero desvinculados de la de prácticas, con lo cual no es necesario realizarlos antes de la misma. Asimismo, los TPR se han diseñado para realizarlos antes de la sesión de prácticas sin ser necesario haber realizado la sesión de problemas. La diferencia entre la secuenciación anterior y la actual se representa en la Figura 1.

Con esta separación se persiguen varios objetivos, en primer lugar, relajar las restricciones de sucesión de actividades de forma que no sea estrictamente necesario realizarlas entre sesiones, evaluar con los EP aspectos más concretos sin estar tan vinculados con un ejemplo real de la práctica que puede hacer más complejo el enunciado de forma innecesaria, y por último mantener el nivel de carga lectiva del trabajo autónomo original.

En concreto, los EP constan de diversas actividades, en forma de problemas, con y sin ayuda 


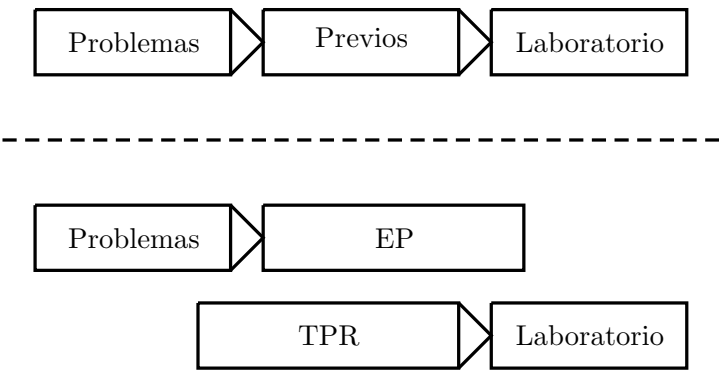

Figura 1: Diferencia entre la secuenciación anterior (arriba) y la actual (abajo).

de ordenador, y actividades usando herramientas desarrolladas en Java para la simulación de sistemas. Algunas de estas actividades son directamente requeridas para evaluar y otras sirven como apoyo para responder a cuestionarios realizados en línea cuyas preguntas se escogen de un banco de preguntas.

Por otra parte, los TPR consisten en el visionado de vídeos y en la propuesta de resolución de ejercicios tipo de las colecciones de problemas. Estas actividades no son evaluadas ya que toda la carga de evaluar la aplicación del conocimiento pasa a los EP, y por lo tanto, se centran más en introducir aspectos más técnicos de la práctica, como el manejo de las herramientas que se van a usar, cómo enfocar los problemas que allí se plantean y su resolución o la relación entre los conceptos teóricos y su repercusión en un entorno práctico; todo ello en formato vídeo. De forma complementaria, se proponen ejercicios tipo para reforzar algunos aspectos en concreto que van a ser tratados en la sesión práctica.

Pese a ser trabajos no evaluados, se ha decidido mantener las tareas previas a las sesiones de laboratorio ya que, desde una concepción constructivista del aprendizaje, según Ausubel [2] si en el sujeto no existen estructuras que le permitan aprender, a las que llama "organizadores previos", hay que crearlas para que el estudiante pueda asimilar los nuevos conocimientos. Esto es lo que se persigue con los TPR, que las sesiones de laboratorio sean más aprovechables para el alumnado.

La carga que suponen los TPR no es muy intensa en comparación a las actividades requeridas en anteriores años antes de las sesiones prácticas, en su lugar, esta carga se ha trasladado a los EP, los cuales mantienen o amplían las tareas a realizar tras la sesión de problemas, de forma que la carga atribuida al trabajo autónomo se mantiene.

Debido a las características de los TPR se ha llevado a cabo un registro de la actividad relacionada con los materiales en concreto, es decir, se ha registrado qué alumno y en qué fecha ha consultado cada uno de los recursos involucrados en cada TPR, sean vídeos o actividades.

Aplicar esta técnica de observación sobre actividades que no van a ser evaluadas, conlleva varias ventajas [9], entre ellas que nos van a permitir describir la conducta de los estudiantes sin el condicionante de la calificación. Además, los datos recopilados sirven de apoyo para observar el comportamiento de los estudiantes cuando son usados en conjunción con otras mediciones, como por ejemplo sus notas. Los datos recopilados mediante este registro de actividad se analizan en la siguiente sección.

\section{RESULTADOS}

La asignatura de Sistemas Automáticos consta de cuatro sesiones de prácticas en laboratorio diferentes. Para el TPR de estas sesiones se han preparado un total de 10 recursos, quedando distribuidos como sigue: Práctica 1, 2 recursos; Práctica 2, 1 recurso; Práctica 3, 4 recursos; y Práctica 4, 3 recursos. Estas 4 prácticas se imparten a 7 grupos de laboratorio distintos, con un total de 156 estudiantes homogéneamente repartidos.

Los recursos usados se presentan en la Tabla 1 donde se ha organizado en cada entrada cada uno de los recursos, indicando a qué práctica pertenecen, el tipo de recurso y se les ha asignado una etiqueta para diferenciarlos.

Entre los TPR de la primera sesión encontramos el vídeo con etiqueta Model_Graf, el cual presenta la resolución de un problema típico sobre modelado con Grafcets, incidiendo en el razonamiento y claves del enunciado que denotan que elementos o estructuras usar. En el vídeo Impl_UPro se muestra como implementar en el programa Unity Pro un diagrama de contactos. Para la segunda sesión, se plantean algunos vídeos Vid_T5, que muestran como identificar, a partir de la respuesta ante escalón, sistemas sin polos ni ceros en el origen y sistemas con integrador. Para preparar la tercera sesión, se plantean los problemas Prob_T7, en los cuales se pide la sintonización de controladores a partir de la respuesta ante escalón conociendo el valor final o de la respuesta con un relé en el bucle. En algunos de estos ejercicios se requiere el uso de la herramienta HJava que permite cargar ficheros con datos experimentales y realizar el ajuste [23]. La resolución de algunos ejercicios de esta colección se muestra en los vídeos Vid_T7. Para ayudar al correcto desarrollo de la sesión, en el vídeo Med_UPro se muestra como registrar, mostrar y hacer mediciones en las señales de entrada de un PLC con Unity Pro. Para la última 


\begin{tabular}{ccccccc} 
Práctica & Etiqueta & Tipo & V. dif AP & V. tot AP & V. dif & V.tot \\
\hline 1 & Model_Graf & Vídeo & 96 & 159 & 101 & 174 \\
1 & Impl_UPro & Vídeo & 90 & 154 & 94 & 165 \\
2 & Vid_T5 & Vídeo & 90 & 192 & 102 & 325 \\
3 & Vid_T7 & Vídeo & 64 & 166 & 84 & 350 \\
3 & HJava & Herramienta & 39 & 57 & 72 & 143 \\
3 & Prob_T7 & Ejercicios & 58 & 115 & 82 & 222 \\
3 & Med_UPro & Vídeo & 73 & 115 & 76 & 118 \\
4 & Vid_T8 & Vídeo & 45 & 94 & 72 & 227 \\
4 & Prob_T8 & Ejercicios & 37 & 68 & 59 & 139 \\
4 & Vid_SisReal & Vídeo & 33 & 40 & 46 & 59
\end{tabular}

Cuadro 1: Recursos para los TPR y sus consultas antes de la práctica (AP) y en todo el periodo.

sesión se plantean los problemas Prob_T8, en los que se pide ajustar controladores PID teniendo el modelo del sistema y cumplir con determinados márgenes de robustez. Algunos de estos problemas se resuelven en los vídeos Vid_T8. Para que los alumnos relacionen todos los conceptos, en el vídeo Vid_SisReal se muestra como los parámetros del controlador afectan y modifican tanto la respuesta en frecuencia como la respuesta temporal del sistema, incidiendo en como modifican los márgenes de robustez y las especificaciones de la respuesta temporal.

Como se ha comentado en el apartado anterior, se ha llevado a cabo un registro de la actividad de cada recurso, quedando registrado quien lo consulta y en qué fecha. Los datos recogidos abarcan fechas entre la activación visible del recurso hasta la fecha del examen final de la primera convocatoria. De esta forma podemos identificar el número de visualizaciones totales por recurso y de visualizaciones de alumnos diferentes por recurso, tanto antes de la fecha de la práctica de cada grupo y antes de la fecha del examen final. Todos estos datos quedan recogidos en la Tabla 1. Las columnas referentes a las visualizaciones recogen aquellas consultas hechas por alumnos diferentes ("dif") y totales, además, el sufijo "AP" indica las consultas realizadas antes de la sesión de prácticas.

A la vista de estos datos se puede observar, en primer lugar, que la participación en la consulta de los recursos no es total por parte de los alumnos. El hecho de que no se evalúe directamente sobre los contenidos de los recursos puede jugar un papel clave. Asimismo, se puede observar una disminución en las consultas a medida que el curso avanza. Muchas pueden ser las causas de este hecho: dificultad de la asignatura, acumulación de tareas o falta de interés en los recursos.

En segundo lugar, que los recursos son consultados en algunos casos más de una vez, lo cual es indicativo del grado de control de los alumnos sobre su educación y de la interacción con su aprendi- zaje. Por otra parte, al dejar los recursos habilitados tras las sesiones, se puede observar que nuevos alumnos visualizan los recursos aún habiendo pasado la sesión para la cual estaban preparados, añadiendo en algunos casos una gran cantidad de consultas totales al recurso. Esto indica que el alumnado percibe una cierta utilidad de los recursos en su formación.

Un caso en concreto para comparar la utilización de los recursos lo encontramos en los recursos relativos a los TPR pertenecientes a la práctica 3 . Los recursos para este TPR están formados por dos vídeos, una colección de ejercicios y de una herramienta informática preparada para trabajar con sistemas simulados. Fijándose en las consultas antes de la sesión de prácticas, se observa una preferencia de los alumnos por consultar los vídeos, aunque haya más de uno, antes que intentar los ejercicios propuestos o trabajar con la herramienta de aprendizaje desarrollada en Java.

Para el estudio de cara al examen final, podemos comparar la colección de problemas Prob_T7 con los recursos de vídeo asociado Vid_T7, los cuales presentan soluciones a algunos problemas de la colección; y la colección de problemas Prob_T8 con algunas de sus soluciones Vid_T8. En estos casos, se puede observar que la consulta de las soluciones en formato vídeo sigue siendo mayor que la consulta de la colección de problemas, tanto en número total de consultas como en alumnos diferentes consultando el recurso. Esto puede conducir a pensar, que parte del alumnado consulta los vídeos como medio para obtener un conocimiento "técnico"de resolución de problemas tipo. En cualquier caso, la máxima diferencia entre la consulta de vídeos y la colección de problemas es de 13 alumnos que apenas representan el $8 \%$ de la muestra.

Además de los datos comentados, se puede obtener también el número total de recursos consultados por cada alumno, tanto antes de la sesión de prácticas como en todo el periodo. Estos datos han sido recopilados y se presentan en la Figura 2, 


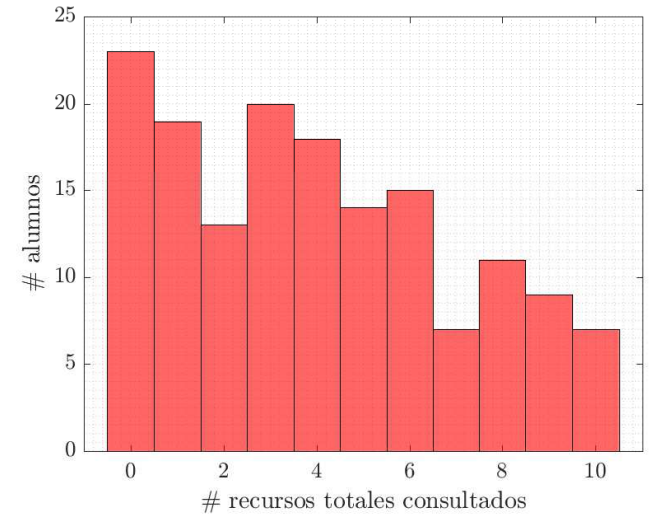

(a) Antes de la práctica.

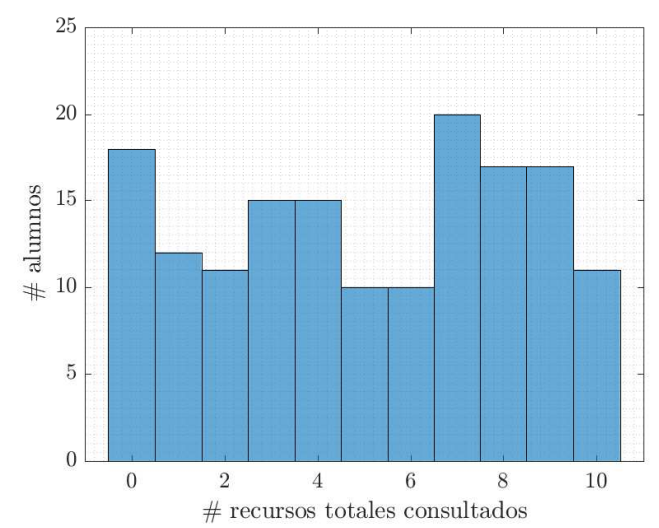

(b) En todo el periodo.

Figura 2: Número de alumnos que han consultado un total de recursos.

donde se presenta una recopilación del número de alumnos que han consultado un número total de recursos, tanto antes de la práctica (Figura 2a), como en todo el periodo (Figura 2b). Los datos reflejan que un tercio del alumnado a consultado más de la mitad de los recursos (suma de barras 6-10 en Figura 2a) pertenecientes a los TPR antes de las sesiones prácticas y de ellos solo 7 alumnos los han consultado todos. En cuanto a los datos presentados para todo el periodo (Figura 2b) se observa cierta evolución, los alumnos han consultado más recursos, aunque solo 4 alumnos se han añadido a la consulta de todos los materiales propuestos.

Con los datos disponibles, es posible agrupar por cada práctica aquellos alumnos que han consultado todo el material recomendable, parte de los recursos y ningún material. Las calificaciones obtenidas por cada uno de los diferentes grupos se presenta en el diagrama de caja de la Figura 3, donde los grupos se marcan con una $T$ para todo el material, $P$ para parte y $N$ para ningún material. Cada sesión de práctica esta separada por una línea vertical.
Con esta representación se puede ver que aparentemente existe cierta correlación entre la consulta de los materiales planteados y el desempeño en las sesiones de laboratorio, aunque esta relación puede no ser causal y sea debida a otros motivos. Si bien no existe una diferencia tan pronunciada entre visualizar la totalidad y visualizar parte del contenido propuesto, sí que se da una mayor diferencia entre estos grupos y no consultar ningún material, tanto en resultados medios como en distribución de los mismos.

Cabe destacar que el volumen de muestras por grupo cambia entre prácticas, siendo los grupos $T$ los más numerosos en las dos primeras prácticas, y los grupos $P$ los más numerosos en las dos últimas, seguidos todos ellos del grupo $N$ haciéndose la diferencia más pequeña con el transcurso de las sesiones.

Finalmente, para poder evaluar si la experiencia docente ha sido satisfactoria se ha incluido una comparativa con las calificaciones obtenidas en cursos anteriores en la Figura 4. En esta figura se comparan las calificaciones obtenidas en el curso 2020/2021 con las obtenidas en los cursos $2017 / 2018,2018 / 2019$ y $2019 / 2020$, ordenadas por prácticas. Se puede observar que los resultados obtenidos en las tres primeras prácticas son bastante similares, siendo incluso ligeramente mejores en las dos primeras prácticas. Sin embargo, en la última práctica los resultados obtenidos son peores en el curso actual que en los anteriores, siendo la mediana de años anteriores superior al corte del primer cuartil de este año. Esta bajada del desempeño puede ser debida a varios factores, como por ejemplo el cansancio acumulado de llevar una docencia a la que los alumnos no están acostumbrados, el incremento de carga asociado al transcurso del curso o la baja tasa de consulta del material asociado a los TPR de esta práctica, consultas que a la vista de la Tabla 1 no llega al $30 \%$ por recurso.

\section{DISCUSIÓN}

El estallido de la pandemia causada por la covid19 ha afectado a todos las facetas de la vida, y entre ellas a la educación. En este trabajo se relatan las decisiones tomadas para seguir ofreciendo una docencia adecuada en el ámbito de la automatización, a pesar de la nueva transición hacia un modelo de educación en línea.

En este contexto, durante este curso se ha decidido reemplazar el trabajo autónomo evaluado a realizar antes de las sesiones prácticas por una serie de trabajos recomendables no evaluados con menor carga lectiva. Esta sustitución viene motivada por 


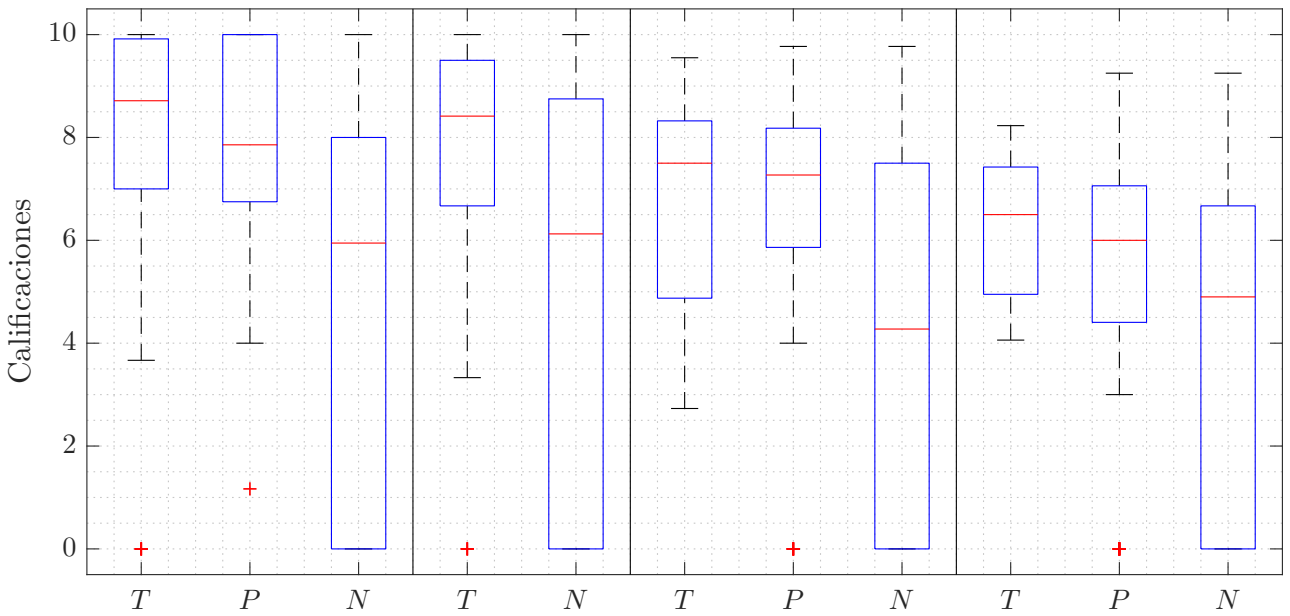

Figura 3: Distribución de las calificaciones obtenidas en las prácticas agrupadas por el grado de consulta de los recursos.

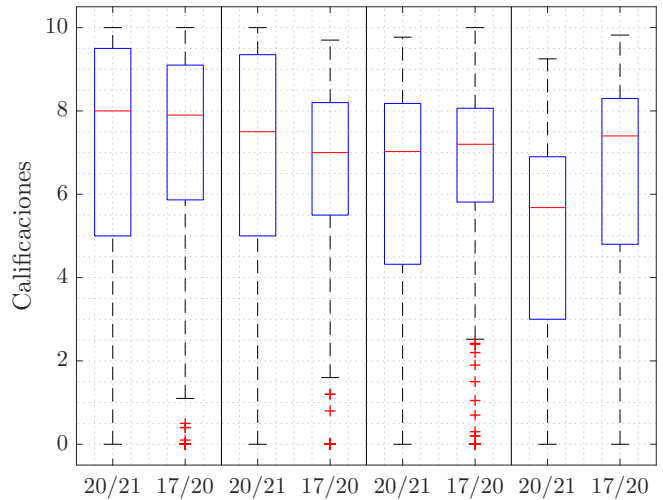

Figura 4: Comparativa de la distribución de calificaciones obtenidas en las prácticas entre el curso $2020 / 2021$ y los cursos 2017/2018-2019/2020.

la falta de disponibilidad de aulas, la cual impide una correcta temporización de la docencia.

Los nuevos contenidos para el trabajo autónomo del alumnado vienen dados en diferentes formatos, entre ellos y el más común en forma de vídeo. Se ha optado por esta vía debido a sus ventajas para el aprendizaje, entre ellas, dotar de más autonomía al alumnado y permitirles regular la carga cognitiva en su aprendizaje.

Para evaluar los efectos producidos por estos cambios se ha llevado a cabo un registro de las consultas realizadas a los recursos propuestos, y se han confrontado estos datos con las calificaciones obtenidas por los estudiantes. En estos datos queda patente la utilidad de los recursos como "organizadores previos"para el aprendizaje, ya que existe cierta relación entre aquellos estudiantes que han consultado los recursos propuestos y las calificaciones más altas.

Se ha observado cierta dejadez por parte de los alumnos en la consulta de los materiales, reduciéndose su consulta un $66 \%$ a lo largo del curso, pudiendo ser causas de la misma el carácter opcional de las tareas propuestas, el aumento de carga de trabajo a lo largo del curso o la adaptación al nuevo modelo de docencia. A consecuencia, las calificaciones obtenidas han ido disminuyendo, quedando patente la relación entre el trabajo previo y el desempeño de los alumnos.

Con respecto a años anteriores, el método propuesto ofrece resultados igualmente favorables, sin embargo, en aquellos casos donde la consulta de los materiales previos ha sido menor, los resultados han sido peores que en otros años.

Por lo tanto, podemos concluir que la experiencia docente ha sido satisfactoria pero mejorable. En primer lugar, nos ha permitido copar con las dificultades derivadas de la ruptura de la temporización. En segundo lugar, los estudiantes han demostrado sacar provecho tanto de las tareas obligatorias empleadas en años anteriores como de los vídeos y materiales opcionales propuestos. Además, en aquellos casos donde se han consultado los materiales propuestos los resultados obtenidos por los alumnos han sido similares a los de otros años.

Sin embargo, queda por mejorar el recalcar a los alumnos la importancia de realizar las tareas propuestas, especialmente a medida que avanza el curso, ya que por diversas causas los alumnos tienden a no tenerlas tan en consideración para su aprendizaje. 


\section{Agradecimientos}

Este trabajo ha sido financiado por la Unitat de Formació $i$ Innovació Educativa de la USE, Universitat Jaume I y por la CEICE número de beca ACIF/2018/244.

\section{English summary}

\section{TEACHING ADAPTATIONS DUE TO COVID-19: EXPERIENCE IN AUTOMATIC ENGINEERING}

\begin{abstract}
This article describes and analyzes the teaching experience related to the academic year 2020-2021 in the subject Automatic systems. Due to the effects of the pandemic, teaching has been adapted since there were not enough classrooms available to allow the correct temporal distribution of the contents. For this reason, new materials have been developed, mostly in the form of video, which allow the follow-up of the course. With the activity records of these new resources, the effectiveness of the method used has been observed, as well as its validity when compared with results from previous years.
\end{abstract}

Keywords: Covid-19, Teaching in engineering, video

\section{Referencias}

[1] Lakmal Abeysekera and Phillip Dawson. Motivation and cognitive load in the flipped classroom: definition, rationale and a call for research. Higher education research $\&$ development, 34(1):1-14, 2015.

[2] David P. Ausubel. The psychology of meaningful verbal learning. 1963.

[3] David P. Ausubel, Joseph D. Novak, Helen Hanesian, et al. Psicología educativa: un punto de vista cognoscitivo, volume 3 . Trillas México, 1976.

[4] Robert M. Bernard, Philip C. Abrami, Eugene Borokhovski, C. Anne Wade, Rana M. Tamim, Michael A. Surkes, and Edward C. Bethel. A meta-analysis of three types of interaction treatments in distance education. Review of Educational research, 79(3):1243-1289, 2009.

[5] Pinaki Chakraborty, Prabhat Mittal, Manu S. Gupta, Savita Yadav, and Anshika Arora. Opinion of students on online education during the covid-19 pandemic. Human Behavior and Emerging Technologies, 2020.

[6] Michelene Chi and Ruth Wylie. The ICAP framework: Linking cognitive engagement to active learning outcomes. Educational psychologist, 49(4):219-243, 2014.

[7] Jennifer Chipps, Petra Brysiewicz, and Maurice Mars. A systematic review of the effectiveness of videoconference-based teleeducation for medical and nursing education. Worldviews on Evidence-Based Nursing, 9(2):78-87, 2012.

[8] Harry de Boer. Covid-19 in dutch higher education. Studies in Higher Education, 46, 2021.

[9] Júlia V. Espín and Mercedes Rodríguez Lajo. L'avaluació dels aprenentatges a la universitat, 1993.

[10] Robert M. Gagné. The Conditions of Learning and Theory of Instruction. Holt, Rinehart and Winston, 1965.

[11] Robert M. Gagné. Principios básicos del aprendizaje para la instrucción. Diana, 1975.

[12] Jimena Gonzalez-Ramirez, Kerri Mulqueen, Ruth Zealand, Sara Silverstein, Christine Mulqueen, and Shawna BuShell. Emergency online learning: College students' perceptions during the covid-19 pandemic. College Student Journal, 55(1):29-46, 2021.

[13] Jisun Jung, Hugo Horta, and Gerard A. Postiglione. Living in uncertainty: the covid19 pandemic and higher education in hong kong. Studies in Higher Education, 46, 2021.

[14] Kyungmee Lee. Coronavirus: universities are shifting classes online-but it's not as easy as it sounds. The Conversation, 9, 2020.

[15] Chi-Sing Li and Beverly Irby. An overview of online education: Attractiveness, benefits, challenges, concerns and recommendations. College Student Journal, 42(2), 2008.

[16] Richard E. Mayer. Applying the science of learning: Evidence-based principles for the design of multimedia instruction. American psychologist, 63(8):760, 2008. 
[17] Barbara Means, Yuki Toyama, Robert Murphy, Marianne Bakia, and Karla Jones. Evaluation of evidence-based practices in online learning: A meta-analysis and review of online learning studies. 2009.

[18] Barbara Means, Yukie Toyama, Robert Murphy, Marianne Baki, et al. The effectiveness of online and blended learning: A metaanalysis of the empirical literature. Teachers College Record, 115(3):1-47, 2013.

[19] Michael Noetel, Shantell Griffith, Oscar Delaney, Taren Sanders, Philip Parker, Borja del Pozo Cruz, and Chris Lonsdale. Video improves learning in higher education: A systematic review. Review of Educational Research, 91(2):204-236, 2021.

[20] Joseph D. Novak. Teoría y práctica de la educación. Ed. Alianza Universidad, 1988.

[21] Charles M. Reigeluth. In search of a better way to organize instruction: The elaboration theory. Journal of instructional Development, pages 8-15, 1979.

[22] Vivien E. Rolfe and Douglas Gray. Are multimedia resources effective in life science education? a meta-analysis. Bioscience education, 18(1):1-14, 2011.

[23] Roberto Sanchis-Llopis. Herramientas de hardware y software libre para la identificación experimental, el diseño y la implementación de controladores PID, 2013.

[24] Sascha Schneider, Steve Nebel, Maik Beege, and Günter Daniel Rey. The autonomyenhancing effects of choice on cognitive load, motivation and learning with digital media. Learning and Instruction, 58:161-172, 2018.

[25] Jo Tomlinson, Tim Shaw, Ana Munro, Ros Johnson, D. Lynne Madden, Rosemary Phillips, and Deborah McGregor. How does telelearning compare with other forms of education delivery? a systematic review of telelearning educational outcomes for health professionals. New South Wales Public Health Bulletin, 24(2):70-75, 2013.

[26] Tamara Van Gog, K. Anders Ericsson, Remy Rikers, and Fred Paas. Instructional design for advanced learners: Establishing connections between the theoretical frameworks of cognitive load and deliberate practice. Educational Technology Research and Development, 53(3):73-81, 2005.
[27] Richard Watermeyer, Tom Crick, Cathryn Knight, and Janet Goodall. Covid-19 and digital disruption in UK universities: afflictions and affordances of emergency online migration. Higher Education, 81, 2021.

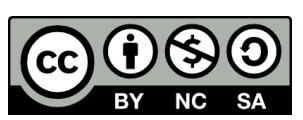

(C) 2021 by the authors. Submitted for possible open access publication under the terms and conditions of the Creative Commons Attribution CC BY-NC-SA 4.0 license (https://creativecommons.org/licenses/by-nc-sa/4.0/deed.es). 